\title{
Building a programming language for a small computer: Reinventing the wheel
}

\author{
GORDON F. PITZ \\ Southern Illinois University, Carbondale, Illinois 62901
}

\begin{abstract}
The language SP-12 for a PDP-12 computer is described. SP-12 is an example of how a special-purpose language can be constructed from a set of machine-language subroutines that are called by a simple interpreter. Symbolic source programs make it easy to learn the language. Preliminary translation of the source program into binary code, using an assembler, greatly increases the speed of the interpreter.
\end{abstract}

In a discussion of pitfalls to avoid in the use of on-line computers, Pilla (1973) cautioned against a user's "reinventing the wheel" by designing special-purpose languages. The present paper is, in a sense, an argument that reinventing the wheel can be a profitable and useful enterprise. It illustrates a solution to problems that arose when I discovered that no one else's wheel would fit my wagon.

When an on-line computer is small, e.g., contains $8 \mathrm{~K}$ or less of core and has no disk unit, the user frequently finds that most programming must be done in machine language. Efficiency of time and space can then become overriding concerns. If higher level interpreters are available, such as FOCAL, they are often intolerably slow and accept only very small programs. Other languages may suffer from one of two faults: (1) because they can be generalized from one machine to another, the languages may not take advantage of the unique facilities of a given small machine; or, (2) the language may be designed for certain types of research (e.g., operant work with animals) and so make other kinds of programming more complex than they need be. This is not to decry the use of existent languages. I merely wish to point out that there may be a need for special-purpose languages, especially with small machines.

Users frequently write subroutines in machine language for most of the operations that are used in day-to-day research. It is a short step to define macro-instructions that call these subroutines; macroinstructions can then form the basis for any special-purpose programming language. Such was the origin of the language SP-12, developed for a PDP-12 computer used in research on decision making and judgment. SP-12 is an attempt to provide for a small 12-bit computer some of the programming power now available for medium-size machines. It is similar to the

Development of SP-12 was supported in part by Grants $G B 28708 X$ and $G B 40313$ from the National Science Foundation and by grants from the Graduate School of Southern Illinois University at Carbondale. Reprint requests should be addressed to G. F. Pitz, Psychology Department, Southern Illinois University at Carbondale, Carbondale, Illinois 62901. SP-12 user's manuals are available on request; source and binary programs for $\mathrm{SP-12}$ can be provided on Linc tape. language HECL, described by Doll (1972), although HECL was developed for a $4 \mathrm{~K}$ machine, and does not include many of the functions available in SP-12. The special-purpose system described by Schneider and Scholz (1973) is a more complex system designed for a PDP-8, but SP.12 was designed with Schneider and Scholz's comments in mind.

\section{DESIGN OF THE LANGUAGE}

Three principle requirements were considered in designing SP-12. First, the language had to be fast enough to handle timing in milliseconds, a speed close to that of assembly language, and there had to be enough space available for reasonably complex programs. Second, the language had to be much easier to learn than assembly language; students generally find it impossible to devote the necessary time to learning PDP-8 and LINC machine-language programming. Third, it was necessary to use LAP-6-DIAL (already in use on the PDP-12) for writing source programs and filing completed programs. The solution to the first problem was to stay close to the concept of an assembly language, avoiding such complexities as nesting and recursion. Ease of programming in SP-12 was improved by avoiding as many syntactic constraints as possible. The third consideration dictated only the general, format of a source program, including labeling and comment statements.

Machine-language subroutines were written to control all peripheral devices attached to the central processor, including the CRT display, magnetic-tape transports, A-D converter, relays, and sense lines. To these routines were added simple arithmetic subroutines to perform single-precision integer addition, subtraction, multiplication, and division. The triple-precision floating-point-arithmetic package provided by Digital Equipment Corporation 1 was included, together with routines necessary to direct the flow of program control. These subroutines form the available functions in SP-12. The functions are listed in Table 1, together with brief descriptions of each.

The limited amount of available core meant that we 
Table 1

SP-12: Summary of Functions

\begin{tabular}{|c|c|}
\hline \multicolumn{2}{|c|}{ Arithmetic Functions } \\
\hline PUT & $\begin{array}{l}\text { Compute value of integer arithmetic expres- } \\
\text { sion and set variable to take on value. }\end{array}$ \\
\hline PUT.F & $\begin{array}{l}\text { Compute value of floating point expression } \\
\text { and set variable to take on value. }\end{array}$ \\
\hline \multicolumn{2}{|c|}{ Transfer Functions } \\
\hline GO & Transfer to program location. \\
\hline DO & Execute subroutine. \\
\hline EXIT & Return from subroutine. \\
\hline \multicolumn{2}{|c|}{ Conditional Transfer Functions } \\
\hline IF & $\begin{array}{l}\text { Transfer contingent upon relation between } \\
\text { integer values or character strings. }\end{array}$ \\
\hline IF.F & $\begin{array}{l}\text { Transfer contingent upon relation between } \\
\text { floating point quantities. }\end{array}$ \\
\hline SWITCH & Transfer contingent upon console switch setting. \\
\hline RESPOND & $\begin{array}{l}\text { Transfer contingent upon occurrence of speci- } \\
\text { fied external response. }\end{array}$ \\
\hline ELAPSE & $\begin{array}{l}\text { Transfer contingent upon elapse of predefined } \\
\text { time interval. }\end{array}$ \\
\hline TRANSFER & $\begin{array}{l}\text { Multiple transfer function: Branch to one of } \\
\text { a list of program labels. }\end{array}$ \\
\hline
\end{tabular}

Iteration Functions

REPEAT Execute program segment for specified values of some variable.

PAUSE Execute program segment for specified time interval.

WAIT Execute program segment until specified response occurs.

Input-Output Functions

INPUT Read in character string or value of variable from Teletype.

CLEAR Clear output buffer of all contents.

OUTPUT Place information in output buffer.

PRINT Print contents of output buffer.

DISPLAY Display contents of output buffer on CRT

PAGE

SIZE

CHANNEL

Move to new page on Teletype.

Specify character size for CRT display.

Specify input-output channel.

DRAW Display values of an array as sequence of points on CRT.

Magnetic Tape Functions

SETUP Establish magnetic tape for data storage.

STORE Store value on magnetic tape.

INDEX Store values on magnetic tape index.

Miscellaneous Functions

START Initiate defined time interval.

DEFINE Define a list of responses to be functionally equivalent.

PRIME Give starting value for random number generator.

PERMUTE Permute values of an array in random order. RELAY CLOSE Execute relay operation.

Terminate program.

had to make a careful choice of functions to be included in SP-12. Potential users were surveyed to discover possible applications of the language. A preliminary version was tested for 18 months to determine the included functions that were not being used, and those useful functions which had not been included. Many operations cannot be carried out conveniently with SP-12; e.g., data stored on magnetic tape cannot be read
Table 2

Special Functions

\begin{tabular}{ll}
\hline ANALOG & Current reading on analog channel. \\
RANDOM & Random number between specified limits. \\
NORMAL & $\begin{array}{l}\text { Normally distributed random number. } \\
\text { REMAINDER }\end{array}$ \\
Remainder resulting from most recent integer \\
division.
\end{tabular}

back for further analysis. But FOCAL can be used for analysis of data collected by an SP-12 program, so we have encountered no difficulties. Given the type of research for which SP-12 is used, the functions now available are probably the most efficient way of using the available space.

A simple executive routine was written that calls the functions in the order given by the user's source program. Each function subroutine is responsible for reading the necessary arguments from the source program. As an example, an integer arithmetic statement in SP-12 might be:

$$
\text { PUT } 45 * X+Y \Rightarrow \text { DATA (I) }
$$

One location is used as an accumulator. The function "PUT" is read by the executive, which calls a subroutine called LOAD. This subroutine calls for a single argument, which is evaluated, and the result (the binary equivalent of 45) is deposited in the accumulator. Control then returns to the executive, which calls the multiplication subroutine. Each arithmetic function calls a single argument, which is added to, subtracted from, multiplies or divides the accumulator, as the case may be. The symbol $\Rightarrow$ identifies a subroutine that calls for a variable. The value of the accumulator is then deposited in the location identified by the name of the variable.

Arguments and variable locations are evaluated by a single subroutine. Arguments may be directly given integers or variables, and variables may have a single subscript. There are also five special functions that provide argument values (see Table 2).

Note that the arithmetic statement specifies the operations to be performed in the order in which they are performed, and this order is always taken from left to right. To compute the value of nested expressions such as $(A+B)^{*}(C+D)$, it is necessary first to evaluate one factor, and then to perform the multiplication, using the statements,

$$
\begin{gathered}
\operatorname{PUT} C+D \Rightarrow X \\
\operatorname{PUT}(A+B) * X \Rightarrow X
\end{gathered}
$$

Parentheses are permitted in a source-program statement, but they are ignored by the interpreter and are used only to improve legibility.

Restricting the arithmetic statements in this way 
makes development of a language much easier, and causes little hardship in the writing of programs for experimental contrcl, as opposed to data analysis. Unlike languages such as FORTRAN, the name of the variable that is to be assigned a value occurs at the end of the statement, not at the beginning, reflecting again the order in which the operations are performed. The arithmetic statement then has a structure very similar to a sequence of instructions in an assembly language.

Because the computer for which SP-12 was written is dedicated to a research program involving experiments in human judgment, there was no need to include provisions for rapid response to interrupt requests. This permitted us to use a simple technique for handling external events. External responses may be switch closures, or information typed at a keyboard. A user may define lists of functionally equivalent responses that are to be detected; e.g., a stimulus display might be terminated when any of a number of switches is pressed. The function WAIT interrogates sequentially each response on the list of equivalent responses. A sequence of program statements (ending with the statement WAITEND) is executed until one of the responses occurs. Programmed time intervals are handled in a similar way; the function PAUSE specifies the duration of the delay in units of $.01 \mathrm{sec}$ or $.001 \mathrm{sec}$, and a sequence of program statements (ending with PAUSEND) is executed until that time period has elapsed. The real-time clock is used only during such delays or in the measurement of response latencies. In the latter case, the clock is set to zero at the appropriate point in the program, and the special function TIME can be used to read the clock at any later point.

The language, then, is strictly linear, with no provision for multiprogram operation or simultaneous handling of multiple inputs or outputs. In this way, less space is used by the interpreter, and more is available for the user's program and data. The inability to run more than one program at a time has not yet proved to be a hardship.

Stimuli generally consist of alphanumeric or point displays on the CRT. The function OUTPUT is used to place information in an output buffer, which can then be displayed or printed. The DRAW function is used to provide a point display of arrays of numerical values. Since the display fades within $30 \mathrm{msec}$ if not refreshed, WAIT or PAUSE functions are used to maintain the display.

The following example is a simple routine that illustrates the handling of stimuli and responses. LIST:1 is a list of possible responses, and $\mathrm{R}$ is the variable used to identify the response that actually occurs.

CLEAR
OUTPUT "RESPOND NOW"
WAIT LIST: 1
DISPLAY
WAITEND $\Rightarrow \mathrm{R}$

\section{CONSTRUCTION OF ASSEMBLER AND INTERPRETER}

The executive routine that calls the sequence of functions in the source program does not refer directly to the symbolic names such as PUT or DATA. As a first step, an assembler converts the symbolic source program into binary code. Each symbolic entry in a source-program statement is translated into a 12-bit number, the value of which indicates the type of entry at that point. For example, all function names are indicated by values between 7725 (octal) and 7777 (octal). If a value outside these limits is encountered where a function is expected, an error message is generated. Similarly, if a number within these limits occurs where an argument is expected, an error message will be generated.

Symbolic labels attached to program statements, and variable names, are identified in the binary object program by the numerical value of the core location at which the statement begins, or where the variable is located. Subscripted variables are handled by adding the value of the subscript to the initial location of the array to give the location of the desired value. The program itself begins at Location 1003 (octal). Unsubscripted variables are located immediately following the program, and arrays of subscripted variables follow the nonsubscripted variables. Hence, one can determine from the location number whether an argument refers to a program label, a nonsubscripted variable, or a subscripted array. Arguments between the limits 772 and 777 are used to identify the special functions. Finally, any argument less than 764 (octal) is assumed to be a literal integer. In other words, integer values can be given directly in a source-program statement, provided they are positive and less than or equal to 500 (decimal).

Negative values, or values greater than 500 , can only be used indirectly. It is possible at the end of a program to define parameters, variables that take on a fixed value. Hence one can use the variable K600, which might be defined as a parameter with the value 600 . Other parameters can be used to set critical values for the experiment. The source program ends with the specification of subscripted arrays. These arrays can be specified by giving their size, or by setting predefined values, as is done with parameters.

The SP-12 system, then, consists of two parts, an assembler that translates the user's source program into binary code, and an interpreter that interprets the binary code. The DIAL monitor permits these two stages to run without interruption so that the user is not aware of the two-stage process. Necessary error checks are included at both stages. The assembler detects only gross syntax errors, such as multiple definitions of symbols, or the use of directly given integers that are too large. Each subroutine in the interpreter contains its own error checks, and the nature of an error can be indicated 
unambiguously by listing the point in the interpreter program at which the error occurs. A debugging routine makes it easy to identify the source-program location that corresponds to any interpreter error. Further details concerning SP-12 are given in the user's manual (Pitz, 1974).

Sp-12 is written for an 8K PDP-12, Version $12 / 30$, with $\mathrm{KW} 12 \mathrm{~A}$ clock. Approximately $5 \mathrm{~K}$ of core is used by the interpreter, leaving the remainder of core storage for the user's program and temporary data storage. Permanent data storage uses the magnetic tapes. This space is adequate for most programs that are likely to be written for a system dedicated to a single research program.

Operation of a program is much faster than can be achieved through most interpreters. For example, it is possible to maintain a $1-\mathrm{msec}$ sampling rate for analog information, at least over a short period of time $(.25 \mathrm{sec})$. A $5-\mathrm{msec}$ sampling rate is possible over extended time periods, when magnetic tape is used to store large quantities of data. Reaction times can be measured in milliseconds. This speed is achieved partly by preliminary translation of symbolic source programs into binary code, and partly by avoiding complex nesting and recurion in defining program statements. An additional time savings is achieved by using two buffers for data to be stored on magnetic tape. A transfer to tape occurs when one buffer is filled, but the use of two buffers means that the tape transfer operation can take place without a pause in the operation of the program.

In summary, SP-12 occupies an intermediate position between assembly language and higher level compliers. For example, a program written in SP-12 will generally be a longer one than an equivalent program written in FOCAL or FORTRAN, but much shorter than the same program written in assembly language. Nevertheless, experience suggests that the language is no more difficult to learn than any high-level language such as FORTRAN.

\section{EXAMPLE}

An example of a program written in SP-12 is given. This is the Sternberg (1966) experiment that seems now to form the benchmark for many program language examples. The form follows that given by Gregg (1973) for APCOL. The program uses 335 (octal) of the 6700 (octal) locations available for a source program.

Certain special characters are used in the source program. Lines and spaces are inserted in the output buffer by means of the symbols ! and $\$$, respectively. Quotation marks enclose a string of text characters to be inserted in the output buffer. A colon is used to link symbolic terms that are combined by the assembler to form a single binary word in the object program; e.g., LINE: 1: ON indicates sense line 1 going to ground. Parentheses, and commas other than those following a statement label, are ignored. Comments begin with a slash mark.

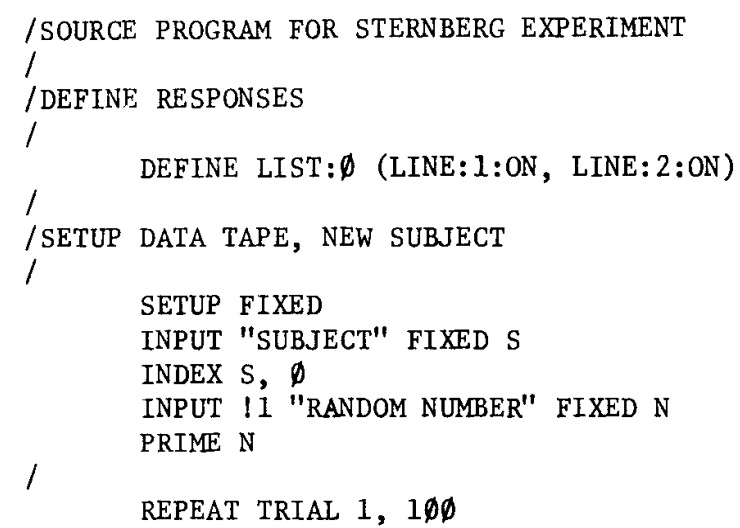


I

IF R NE CORRECT, GO WRONG OUTPUT ! $2 \$ 6$ "CORRECT" GO FEEDBK

WRONG, OUTPUT! 2 \$7 "WRONG"

I

FEEDBK, PAUSE $3 \emptyset \emptyset$ CSEC

DISPLAY

PAUSEND

$/$

/END OF TRIAL: STORE DATA

I

STORE PSET

STORE PROBE

STORE R

STORE LATENCY

/

END

CLOSE

\section{$/$}

ARRAYS

NUMBER, $\varnothing 12234456789$

1

/END OF STERNBERG PROGRAM

\section{REFERENCES}

Doll, T. J. A 4-K computer language for experimentation with human subjects. Behavior Research Methods \& Instrumentation, 1972, 4, 23-33.

Gregg, L. W. APCOL: An example. Behavior Research Methods \& Instrumentation, $1973,5,248$

Pilla, M. A. Co-existing with on-line systems. Behavior Research Methods \& Instrumentation, 1973, 5, 80-82.

Pitz, G. F. SP.12 programmer's reference manual. Psychology Department, Southern Illinois University at Carbondale, 1974.

Schneider, W., \& Scholz, K. W. Requirements for mini computer operating systems for human experimentation and an implementation on a 4-K PDP-8 computer. Behavior Research Methods \& Instrumentation, 1973, 5, 173-177.

Sternberg, S. High-speed scanning in human memory, Science, $1966,153,652.654$.

\section{NOTE}

1. See Document DEC-YOYB-D, published by Digital Equipment Corporation.

(Received for publication October 11, 1974; revision received November 25,1974 .) 